\title{
Aortic Arch Quantification using Efficient Joint Segmentation and Registration
}

\author{
Andreas Biesdorf ${ }^{1}$, Karl Rohr ${ }^{1}$, Hendrik von Tengg-Kobligk ${ }^{2}$, Stefan Wörz ${ }^{1}$ \\ ${ }^{1}$ University of Heidelberg, BIOQUANT, IPMB, and DKFZ Heidelberg \\ Dept. Bioinformatics and Functional Genomics, Biomedical Computer Vision Group \\ ${ }^{2}$ University Hospital Heidelberg, Dept. of Diagnostic and Interventional Radiology \\ a.biesdorf@dkfz.de
}

\begin{abstract}
Accurate aortic arch quantification is important for diagnosis and treatment of cardiovascular diseases. We introduce a new approach for the quantification of the aortic arch morphology with improved computational efficiency which combines 3D model-based segmentation with intensity-based image registration. The performance of the approach has been evaluated based on 3D synthetic images and clinically relevant 3D CTA images including pathologies. We also performed a quantitative comparison with a previous approach.
\end{abstract}

\section{Introduction}

Accurate segmentation of the aortic arch is crucial for diagnosis and treatment of cardiovascular diseases. Pathologies of the aortic arch may be treated by minimally-invasive placement using an endovascular graft, which should be chosen based on the anatomy of each patient. Therefore, individual morphological parameters such as the centerline position and the vessel diameters have to be quantified. The geometry of the aortic arch can be automatically determined from radiological images by segmentation approaches such as, for example, region growing, differential approaches, or deformable models.

Recently, increased attention has been paid to combined approaches that integrate segmentation and registration. These approaches can be classified as model-to-image registration (e.g., $[1,2,3])$ or as joint segmentation and registration approaches (e.g., [4, 5, 6]). While in model-to-image registration, segmentation is performed by image registration of a model, joint approaches combine segmentation and registration in a single functional. Joint approaches for the segmentation of human vessels have only recently been suggested [6].

In this contribution, we introduce a novel joint segmentation and registration approach for the quantification of the aortic arch morphology from 3D tomographic images.

\section{Materials and Methods}

Our approach for the segmentation of vessels in 3D tomographic images combines model-based segmentation with elastic image registration. The approach is based 
on an energy-minimizing functional $J_{k}$ corresponding to a vessel segment $k$

$$
J_{k}\left(p_{k}, u_{k}\right)=J_{M}\left(g_{M}, g_{I, k}^{\mathrm{roi}}, p_{k}\right)+J_{R}\left(g_{I, k}^{\mathrm{roi}}, g_{M, k}^{\mathrm{roi}}, u_{k}\right)
$$

The first term $J_{M}$ denotes an intensity similarity measure between a 3D cylindrical intensity model $g_{M}$ with parameters $p_{k}$ and the intensities $g_{I, k}^{\text {roi }}$ within a region-of-interest (ROI) of a $3 \mathrm{D}$ tomographic image $g_{I}$. The second term $J_{R}$ denotes an energy-minimizing functional for elastic registration of $g_{I, k}^{\text {roi }}$ with an image $g_{M, k}^{\text {roi }}$ generated from the 3D intensity model $g_{M}$.

The 3D parametric intensity model used in $J_{M}$ represents an ideal sharp 3D cylinder convolved with a 3D Gaussian [7]. The model includes parameters for the width $R$ of the tubular structure and the image blur $\sigma$, and is well-suited to describe the plateau-like intensity structure of thick vessels. The complete 3D parametric intensity model $g_{M}$ also incorporates intensity levels $a_{0}$ (surrounding tissue) and $a_{1}$ (vessel) as well as a $3 \mathrm{D}$ rigid transform $\mathcal{R}$ with rotation $\boldsymbol{\alpha}=$ $(\alpha, \beta, \gamma)^{T}$ and translation $x_{0}=\left(x_{0}, y_{0}, z_{0}\right)^{T}$, which yields $g_{M}(x, p)=a_{0}+\left(a_{1}-\right.$ $\left.a_{0}\right) g_{C y l}\left(\mathcal{R}\left(x, \boldsymbol{\alpha}, x_{0}\right), R, \sigma\right)$ with parameters $p=\left(R, a_{0}, a_{1}, \sigma, \alpha, \beta, \gamma, x_{0}, y_{0}, z_{0}\right)^{T}$.

The cylindrical model $g_{M}$ can accurately represent a vessel segment if the vessel has circular cross-sections. However, the model may be inaccurate in the case of non-circular cross-sections (e.g., Fig. 1a). To improve the accuracy between the model and the true vessel shape in this case, we suggest using elastic registration of an image $g_{M, k}^{\text {roi }}$ generated from the $3 \mathrm{D}$ intensity model $g_{M}$ with the original image $g_{I, k}^{\text {roi }}$. The result of elastic registration is a deformation field $u_{k}$ which is used to compute a refined vessel contour and centerline position.

The functional in (1) is optimized by an iterative scheme which alternatingly minimizes $J_{M}$ and $J_{R}$ for each vessel segment $k$ to obtain estimates for the model parameters $p_{k}$ and the deformation field $u_{k}$. For a vessel segment $k$, we estimate $p_{k}$ by least-squares model fitting of $g_{M}$ to the image intensities $g_{I, k}^{\text {roi }}$. To compute $u_{k}$, we generate an image $g_{M, k}^{\text {roi }}$ using the fitted intensity model $g_{M}$ and perform intensity-based registration with $g_{I, k}^{\text {roi }}$ by minimizing $J_{R}\left(u_{k}\right)=J_{\text {Data }, I}\left(g_{I, k}^{\text {roi }}, g_{M, k}^{\text {roi }}, u_{k}^{I}\right)+\lambda_{I} J_{I}\left(u_{k}, u_{k}^{I}\right)+\lambda_{E} J_{\text {Elastic }}\left(u_{k}\right)$, where $\lambda_{I}$ and $\lambda_{E}$ are scalar weights. The first term $J_{\text {Data }, I}$ describes the intensity-based similarity measure between $g_{I, k}^{\text {roi }}$ and $g_{M, k}^{\text {roi }}$ (sum-of-squared intensity differences). With the second term $J_{I}$, the intensity-based deformation field $u_{k}^{I}$ is coupled with the final deformation field $u_{k}$ using a weighted Euclidean distance. The third term $J_{\text {Elastic }}$ represents the regularization of the deformation field according to the Navier equation of linear elasticity

The result of elastic registration is used to improve the result of model fitting by re-estimating the model parameters $p_{k}$ including the radius $R$, the orientation $\boldsymbol{\alpha}$, as well as the translation $x_{0}$. Based on the updated parameter vector $p_{k}$ and the deformation field $u_{k}$, we again perform model-based segmentation with subsequent elastic registration for minimizing $J$. This alternating optimization is repeated until the results of model fitting and elastic registration converge for a vessel segment $k$. After convergence and having estimated the parameters for the current vessel segment, a new parameter vector $p_{k+1}$ is predicted based on a Kalman filter and used as initialization for the next vessel segment. 
We have developed two different variants of our approach to exploit the intensity information. The first variant performs model fitting within a 3D ROI and uses $3 \mathrm{D}$ image registration within the $3 \mathrm{D}$ ROI. The second variant uses 3D model fitting only for estimating the initial 3D orientation $\boldsymbol{\alpha}$, while $J_{k}$ is minimized based on model fitting and image registration of $2 \mathrm{D}$ image crosssections orthogonal to the vessel centerline.

To reduce the computational complexity of our approach, we introduce an automatic adaptive masking scheme. The idea is to perform intensity-based registration not for the whole ROI but only for those regions which contain relevant information. In our application, most information is contained in edge regions of a vessel. Hence, in each iteration $k$ of the segmentation we generate a binary mask

$$
m_{k}^{\text {roi }}(x)=\left\{\begin{array}{l}
1, \text { if }\left\|\nabla g_{M, k}^{\mathrm{roi}}(x)\right\|>c_{t} \cdot \max _{x \in R O I}\left(\left\|\nabla g_{M, k}^{\mathrm{roi}}(x)\right\|\right) \\
0, \text { otherwise }
\end{array}\right.
$$

where $\|\cdot\|$ denotes the Euclidean norm, and $\max _{x \in R O I}\left(\left\|\nabla g_{M, k}^{\text {roi }}(x)\right\|\right)$ denotes the maximum magnitude of the gradient of $g_{M, k}^{\text {roi }}$ within the ROI (we used $c_{t}=$ 0.1). Note that the gradient is computed based on the model, i.e., noise and neighboring structures in the original image do not disturb the result.

\section{Results}

We have applied our approach to 120 3D synthetic images and 15 clinically relevant 3D CTA images of the human thorax. To quantify the segmentation accuracy, we have computed mean errors for clinically relevant measures comprising the minimum, mean, and maximum vessel diameters, $\bar{e}_{D \text {,min }}, \bar{e}_{D \text {,mean }}$, and $\bar{e}_{D, \max }$, respectively, and the mean error for the centerline position $\bar{e}_{x_{0}}$.

In a first experiment, we have generated 120 images of twisted tori with elliptical cross-sections that differ in radii and the level of Gaussian image noise (see

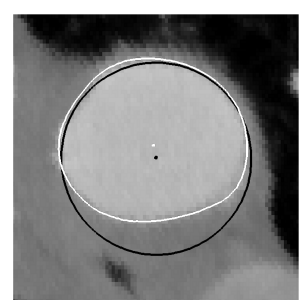

(a)

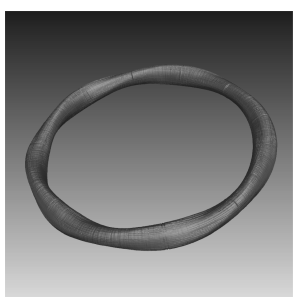

(b)

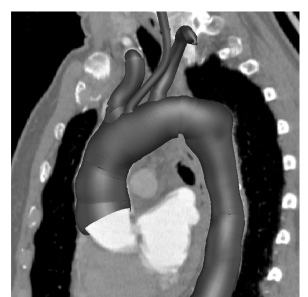

(c)

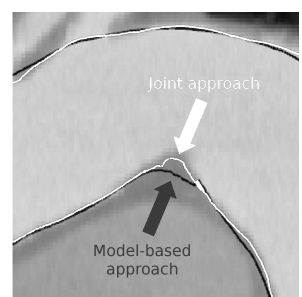

(d)

Fig. 1. (a) Cross-section of a 3D CTA image of an aorta and overlaid result of the model-based (black) and 3D joint approach (white). (b) Segmentation result of the 2D joint approach for a 3D synthetic image of a twisted cylinder. (c) Segmentation result of the 3D joint approach for a 3D CTA image. (d) Computed vessel contours using the model-based (black) and 3D joint approach (white) for a section of a 3D CTA image. 
Fig. 1b). We have evaluated the segmentation accuracy of the new joint approach (2D and 3D variant) in comparison to a previous model-based approach [7]. The previous approach yields $\bar{e}_{D, \text { min }}=3.12$ voxels, $\bar{e}_{D \text {,mean }}=0.11$ voxels, and $\bar{e}_{D, \max }=3.85$ voxels. For the $2 \mathrm{D}$ joint approach we obtain $\bar{e}_{D, \min }=1.05$ voxels, $\bar{e}_{D, \text { mean }}=0.09$ voxels, and $\bar{e}_{D, \text { max }}=1.52$ voxels, which is a significant improvement for the minimum and maximum diameters, while for $\bar{e}_{D \text {,mean }}$ we obtain a similar result. For the $3 \mathrm{D}$ joint approach, we obtain $\bar{e}_{D, \text { min }}=0.53$ voxels, $\bar{e}_{D \text {,mean }}=0.16$ voxels, and $\bar{e}_{D, \max }=0.47$ voxels, which is the best result for the minimum and maximum diameters. For $\bar{e}_{x_{0}}$, the previous approach yields $\bar{e}_{x_{0}}=0.16$ voxels, while we obtain improved results for the new $2 \mathrm{D}$ and $3 \mathrm{D}$ approaches with $\bar{e}_{x_{0}}=0.11$ voxels and $\bar{e}_{x_{0}}=0.09$ voxels, respectively. It also turned out that the adaptive masking scheme reduces the computation time by $32 \%$ for the $3 \mathrm{D}$ approach and by $13 \%$ for the $2 \mathrm{D}$ approach.

In a second experiment, we applied our approach to two different sets of 3D CTA images of the thorax. The first set of images contains ten 3D CTA images of patients with only slight pathologies (see Fig. 1c,d). The second set of images contains five 3D CTA images of patients with severe pathologies (see Fig. 2). It turned out that for $\bar{e}_{D, \min }$ and $\bar{e}_{D, \max }$ the new 2D and 3D approaches yield more accurate results than the previous approach. For $\bar{e}_{x_{0}}$, the 2D approach yields the best result, while for $\bar{e}_{D \text {,mean }}$, the $3 \mathrm{D}$ approach yields the best result.

In addition, we applied our approach to pathological vessels in five different 3D CTA images. It turned out that the new approach significantly decreases the computation time by $41 \%$ for the $3 \mathrm{D}$ variant and by $28 \%$ for the $2 \mathrm{D}$ variant (see Table 1). For $\bar{e}_{D, \text { min }}$ and $\bar{e}_{D, \max }$, the segmentation accuracy of the $2 \mathrm{D}$ approach is slightly reduced, while for the $3 \mathrm{D}$ approach, similar results are obtained. For $\bar{e}_{D \text {,mean }}$ and $\bar{e}_{x_{0}}$ both the new 2D and 3D approaches improve the segmentation accuracy in comparison to the unmasked approach. Moreover, for the clinically most relevant diameter measures the new approach consistently yields significant improvements compared to the previous pure model-based approach.

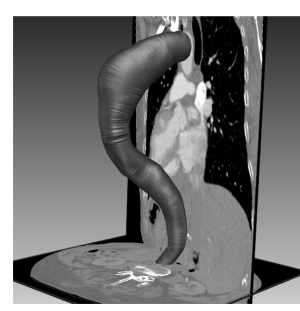

(a)

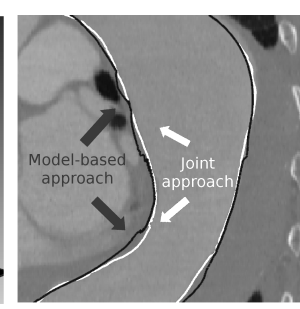

(b)

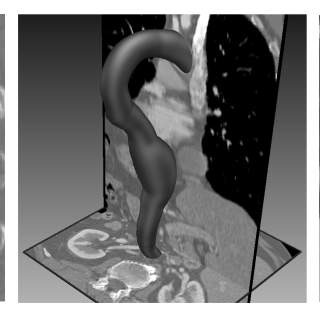

(c)

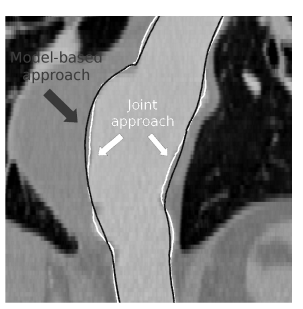

(d)

Fig. 2. (a), (c) Segmentation results of the 3D joint approach for two 3D CTA images showing a pathology. (b), (d) Vessel contours using the model-based approach (black) and the 3D joint approach (white) for a section of the 3D CTA images. 
Table 1. Mean errors for the diameters $\bar{e}_{D, \text { min }}, \bar{e}_{D \text {,mean }}$, and $\bar{e}_{D \text {,max }}$, and the centerline position $\bar{e}_{x_{0}}$ for different approaches as well as the mean computation time $\bar{t} /$ seg for the unmasked (U) and adaptive masking (M) approach.

\begin{tabular}{lrrrrrrrrrrr}
\hline & \multicolumn{1}{c}{$\bar{e}_{D, \min }$} & \multicolumn{2}{c}{$\bar{e}_{D, \text { mean }}$} & \multicolumn{2}{c}{$\bar{e}_{D, \max }$} & \multicolumn{3}{c}{$\bar{e}_{x_{0}}$} & \multicolumn{2}{c}{$\bar{t} /$ seg $(\mathrm{sec})$} \\
Approach & $\mathrm{U}$ & $\mathrm{M}$ & $\mathrm{U}$ & $\mathrm{M}$ & $\mathrm{U}$ & $\mathrm{M}$ & $\mathrm{U}$ & $\mathrm{M}$ & $\mathrm{U}$ & $\mathrm{M}$ \\
\hline Model-based approach & 5.11 & \multicolumn{1}{c}{1.56} & \multicolumn{2}{c}{3.14} & \multicolumn{2}{c}{0.80} & \multicolumn{2}{c}{0.33} \\
2D joint approach & 2.60 & 2.94 & 1.40 & 1.26 & 1.54 & 1.85 & 0.63 & 0.60 & 1.37 & 0.98 \\
3D joint approach & 2.19 & 2.13 & 1.23 & 1.12 & 1.40 & 1.43 & 1.13 & 0.92 & 32.80 & 19.20 \\
\hline
\end{tabular}

\section{Discussion}

We have introduced a new joint approach for the quantification of the aortic arch from 3D CTA images that combines fitting of a parametric intensity model with intensity-based elastic image registration. We have demonstrated the applicability of our approach using 3D synthetic images and clinically relevant 3D CTA images. From the experiments it turned out that the new approach consistently yields more accurate segmentation results than a previous pure model-based approach for the minimum and maximum diameters. It also turned out that for the new approach significant improvements are obtained for difficult segmentation tasks, in particular, for pathologies. Furthermore, the new joint approach with the adaptive masking scheme leads to a significant reduction in computation time while the segmentation accuracy remains similar for the minimum and maximum diameters and the accuracy is even increased for the mean diameter.

\section{References}

1. Aylward SR, Jomier J, Weeks S, et al. Registration and analysis of vascular images. Int J Computer Vis. 2003;55(2/3):123-38.

2. Groher M, Zikic D, Navab N. Deformable 2D-3D registration of vascular structures in a one view scenario. IEEE Trans Med Imaging. 2009;28(6):847-60.

3. Isgum I, Staring M, Rutten A, et al. Multi-atlas-based segmentation with local decision fusion: application to cardiac and aortic segmentation in CT scans. IEEE Trans Med Imaging. 2009;28(7):1000-10.

4. Yezzi A, Zollei L, Kapur T. A variational framework for joint segmentation and registration. In: Proc IEEE Comput Soc Workshop Math Methods Biomed Image Anal. Kauai, HI/USA; 2001. p. 44-51.

5. Schmidt-Richberg A, Handels H, Ehrhardt J. Integrated segmentation and nonlinear registration for organ segmentation and motion field estimation in 4D CT data. Methods Inf Med. 2009;48(4):344-49.

6. Biesdorf A, Rohr K, von Tengg Kobligk H, et al. Combined model-based segmentation and elastic registration for accurate quantification of the aortic arch. Proc MICCAI. 2010; p. 444-51.

7. Wörz S, Rohr K. Segmentation and quantification of human vessels using a 3D cylindrical intensity model. IEEE Trans Image Process. 2007;16(8):1994-2004. 